\title{
DOE ER63951-3 Final Report: An Integrated Assessment of Geochemical and Community Structure Determinants of Metal Reduction Rates in Subsurface Sediments
}

\begin{abstract}
The objective of this research was to examine the importance of microbial community structure in influencing uranium reduction rates in subsurface sediments. If the redox state alone is the key to metal reduction, then any organisms that can utilize the oxygen and nitrate in the subsurface can change the geochemical conditions so metal reduction becomes an energetically favored reaction. Thus, community structure would not be critical in determining rates or extent of metal reduction unless community structure influenced the rate of change in redox.

Alternatively, some microbes may directly catalyze metal reduction (e.g., specifically reduce U). In this case the composition of the community may be more important and specific types of electron donors may promote the production of communities that are more adept at U reduction. Our results helped determine if the type of electron donor or the preexisting community is important in the bioremediation of metal-contaminated environments subjected to biostimulation.

In a series of experiments at the DOE FRC site in Oak Ridge we have consistently shown that all substrates promoted nitrate reduction, while glucose, ethanol, and acetate always promoted $U$ reduction. Methanol only occasionally promoted extensive $U$ reduction which is possibly due to community heterogeneity. There appeared to be limitations imposed on the community related to some substrates (e.g. methanol and pyruvate). Membrane lipid analyses (phospholipids and respiratory quinones) indicated different communities depending on electron donor used. Terminal restriction fragment length polymorphism and clone libraries indicated distinct differences among communities even in treatments that promoted $U$ reduction. Thus, there was enough metabolic diversity to accommodate many different electron donors resulting in the U bioimmobilization.
\end{abstract}

\section{Overview:}

This research project represented a joint effort between Oak Ridge National Laboratory (ORNL), the University of Tennessee (UT) and Florida State University (FSU). ORNL served the lead institution and was responsible for project coordination, integration, and deliverables. This project funded two years of research. The overall goal of this project was to provide an improved understanding of the relationships between microbial community structure, geochemistry, and metal reduction rates.

This work was conducted with subsurface materials collected from the Oak Ridge Integrated Field Challenge (ORIFC) established by the DOE-ERSP program in Oak Ridge, Tennessee. The primary objective of year 1 under Task 1 was the development and optimization of microcosm experiments designed to manipulate uranium reduction activity. Under Task 2 in years 1 and 2, we proposed to further define experimental conditions for the perturbation of microbial community structure by manipulating electron donor, electron shuttle, and nutrient concentrations. Five microcosm experiments were conducted. Results from these experiments were submitted as abstracts to the American Society for Microbiology International Annual Meeting and the International Conference on Bioinformatics \& Computational Biology. The combination of experiments has been processed into peer-reviewed manuscripts. Summary results are provided below. A listing of publications and presentations delivered follows the summary of results. 


\section{Summary of Results:}

In order to provide a further understanding of the coupled microbiological and geochemical processes limiting U(VI) immobilization, we determined the rates of nitrate and uranium reduction and the changes in microbial community composition when different electron donor were used in microcosm experiments. The microcosm experiments used ORIFC subsurface sediments and groundwater close to in situ conditions.

Three microcosm experiments (identified as 3,5 and 8) were constructed with sediments from Area 2 that were homogenized under anaerobic conditions in the FRC glove box. Each microcosm consisted of 20 grams of sediment with $80 \mathrm{~mL}$ site groundwater from a standard on site well in a $250 \mathrm{~mL}$ Pyrex bottle capped with butyl rubber stoppers that contain an anaerobic pressure tube sampling port. For experiments 3 and 5, methanol [40mM] (+/- humics [30 ppm]), ethanol $[20 \mathrm{mM}]$, pyruvate $[24 \mathrm{mM}]$, glucose $[10 \mathrm{mM}]$, and a control with no added electron donor were the carbon sources used in the experiments. The concentrations were adjusted to equal the electron potentials of the donor. The $\mathrm{pH}$ was adjusted to 7.0 with sodium bicarbonate for experiments 5 and 8 and in experiment 3, methanol and ethanol were also tested over four $\mathrm{pH}$ values of 5.5, 6.0, 6.5, and 7.0. For experiment 8 , spatial heterogeneity was examined using $1 \mathrm{~m}$ interval cores (FWB120) at depths starting at 4.0, 7.0, 8.2, 8.8 and $12.5 \mathrm{~m}$ and using groundwater came from area 2: FW209. Methanol $[40 \mathrm{mM}]$, ethanol $[20 \mathrm{mM}]$, ethanol $[10 \mathrm{mM}]$ with pyruvate $[12 \mathrm{mM}]$, glucose $[10 \mathrm{mM}]$, and a control with no added electron donor were the carbon sources used in the experiments. The microcosms were performed in triplicate or quadruplet. Microcosms were sampled for membrane lipid analyses at the first and last sampling time point. Sediment slurry aliquots were placed in Whirl pak ${ }^{\mathrm{TM}}$ bags and frozen at $-80^{\circ} \mathrm{C}$ and then lyophilized prior to membrane lipid analyses. During the time course experiment aliquot samples were analyzed for nitrate and uranium reductions. The HACH method was used to measured nitrate spectrophotometerically. A Chemchek KPA (kinetic phosphorescence analyzer) was used to measure the uranium in diluted samples.

Results from Experiment 3 showed that nitrate reduction was rapid and that differences among substrates were small. The methanol showed a lag time while glucose, ethanol, and lactate did not. There was minimal to no effect of $\mathrm{pH}$. Uranium reduction showed initial increases which could be related to kinetic effects on equilibrium in slurries, reoxidation due to nitrate, or leakage of air into microcosms. No reduction of uranium was observed in the control or methanol treatment. Pyruvate starts very high and continues to increase. For experiment 5 the nitrate and $U$ reduction data was similar. The one exception was that methanol amended microcosms in experiment 5 did reduce uranium. For methanol-amended microcosms, iron reduction occurred concurrently with $U$ reduction and was visually assessed by color changes in the sediments. For experiment 8 , nitrate, sulfate and uranium reduction were monitored. Site heterogeneity was seen in the starting concentration of each of these electron acceptors with nitrate ranging from 51 to $78 \mathrm{ppm}$, sulfate ranging from 2 to $22 \mathrm{ppm}$, and uranium ranging from 0.06 to $5.37 \mathrm{ppm}$. Nitrate reduction was minimal expect for the ethanol with pyruvate treatment at depths 7 and $8.8 \mathrm{~m}$. Uranium was reduction at depths $4,8.2$, and $12.5 \mathrm{~m}$ in the ethanol with pyruvate microcosms. Sulfate was not reduced and at a couple of depths there was a slight increase is sulfate concentrations compared to $t=0$.

Biomass as estimated by phospholipid fatty acid methyl ester (PLFAME) analyses ranged from approximately 10 to 30,000 pmol g-1 dry weight among all the samples. Bacterial cell numbers from the recovered PLFA were estimated to range from $2.5 \times 10^{5}$ to $7.5 \times 10^{8}$ cells g $^{-1}$ (Balkwill et al., 1988). The control microcosms general had a lower biomass than the electron 
donor amended microcosms. The exception was seen at the $12.5 \mathrm{~m}$ depth in experiment 8 where the methanol addition had lower biomass that the control samples for that depth. Microcosms supplemented with electron donor and at $\mathrm{pH} 7$ showed a 10- to 20-fold increased microbial biomass compared to microcosms without electron donor addition. Methanol amended microcosms at lower $\mathrm{pH}$ conditions were not significantly different from the control samples. However the ethanol treatment at $\mathrm{pH} 5$ showed biomass estimates similar the ethanol treatment at $\mathrm{pH} 7.0$.

The microbial community PLFAME compositions varied based on the electron donor

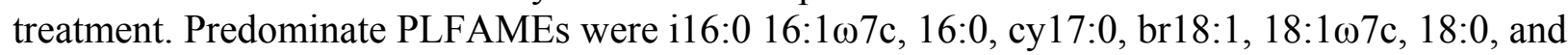
cy19:0. Compared to the PLFAME profile for the control microcosms, the substrate additions resulted in increases in monounsaturates which are indicative of gram negative bacteria. With the exception of 3-M 5.0, the methanol microcosms had a community dominated by 16 carbon fatty acids, whereas the glucose, ethanol and pyruvate microcosms had a mix of 16 and 18carbon fatty acids. The methanol with humic amendment shared the largest relative proportions of iso- and antiiso-17:0 and 10Me16:0, which are associated with sulfate and metal reducers, such as Desulfomonas, and other anaerobic prokaryotes. The glucose amendments show the largest relative proportions of iso and antiiso 15:0 and iso16:0, which are associated with gram $^{+}$ bacteria such as Clostridia and other anaerobic bacteria.

Ratios of specific PLFAME biomarkers are used to provide insight into the nutritional and physiological status of the microbial community. Interestingly, a considerable percentage of the total PLFAME profiles, with as much as 35\% in some microcosms, were identified as cy17:0. With the elevated levels of cyclopropyl fatty acids, the cyclopropyl to monounsatruate ratio was examined to see if nutritional stress was indicated. Experiment 8 microcosms with methanol amendments had 5 times higher ratio values than the values observed for ethanol or pryruvate amended microcosms. Likewise elevated cyclopropyl to monousaturate ratios were seen for some controls in experiment 3 and 8. In the low biomass samples this ratio indicated nutristional limitations while in higher biomass samples the elevated levels of cy17:0 was associated with sulfate and metal-reducing bacteria and other anaerobic bacteria. Methanol amendments for experiments 3, 5 and 8, control depths samples at 8.8 and $12.5 \mathrm{~m}$ for experiment 8 , and the $\mathrm{pH} 7.0$ ethanol amendments for experiments 3 and 5 had trans to cis PLFAME ratios that indicated toxic or limiting conditions.

Given the complexity of these PLFAME profiles, a hierarchical cluster analysis was performed to show relatedness between samples in experiment 3 and 5. The dendrogram (single linkage, euclidean distance) showed clusters by experiment which may be explained by site heterogeneity. Within experiment 3, the low $\mathrm{pH}$ methanol amended microcosms clustered separately for the other treatments. Whereas experiment 5 had two main clusters with the first cluster contain the control and methanol amended microcosms as separate branches within the cluster and the second cluster containing ethanol and glucose amended microcosms.

The PLFAME community compositions correspond with the clone distributions for methanol and methanol with humic samples that showed uranium reduction. Many experiment 5 clones were associated with organics (e.g. solvent, toluene) degradation as represented by Trichlorobacteria (delta-proteoacteria) and Polaromonas (beta-proteobacteria). Another dominant beta-proteobacteria was Rhodoferax. Compared to non-U-reducing methanol microcosms, the methanol microcosms with $U$ reduction had more delta-proteobacteria (e.g. Geobacter), Sphingo-bacteria (e.g. Roseivirga), and Firmicutes (e.g. Clostridia). 
Membrane lipids were also investigated for respiratory quinone composition. The quinone profile consisted of ubiquinones (UQ) and menaquinones (MK) with varying isoprene units on the side chain. Ubiquinones are produced by gram bacteria grown under aerobic conditions. Menaquinones are produced by gram ${ }^{-}$bacteria grown under anaerobic conditions, gram $^{+}$bacteria and archaea. For experiments 3 and 5, MK7 was the more prominent quinone for the methanol and glucose treatments, while MK8 was more prominent for the pyruvate, ethanol and control treatments. MK6 represented $10 \%$ of the quinone profile in the control microcosms followed by $10 \%$ in the glucose and pyruvate amended microcosms. Different respiratory quinones were observed in experiment 8. MK7 and MK4 dominated the ethanol amended microcosms, while MK4 and MK6 dominated the methanol amended microcosms. These shifts in respiratory quinones suggest changes within in the microbial communities' present under the respective conditions of the different experiments.

In experiments 3 and 5 the UQ to MK ratio ranged from 0.00 to 0.05 , which indicated the microcosms at the final time point had anaerobic conditions. Two samples containing glucose were exceptions with UQ/MK ratio of 0.25 to 1.00 and were suspected of having leaks resulting in oxygen exposure. In experiment 8 the UQ to MK ratios were more varied. Nine samples were in the 0.00 to 0.15 range indicating anaerobic condition, while three samples were in the 0.20 to 0.40 range, which indicated some recent exposure to air. Four samples had ratios at the 0.70 to 1.00 range indicating aerobic conditions. It appears that several of the microcosms for experiment 8 at the $12.5 \mathrm{~m}$ depth may have had leaks and this could easily explain why uranium reduction was not seen in most of the microcosms.

Several bacteria produce MK7 including Bacillus, Clostridium, and Desulfotomaculum. Desulfovibrio produces MK6. Gram-negative facultative anaerobic rods produce MK8, but they also produce demethylmenaquinones that were not detected in these microcosms. Arthrobacter, Breviobacterium and Corynebacterium have either MK8 or MK9, while Nocardia and Rhodocuccus have MK8. When considering this brief listing of organisms and quinones with the microcosm analysis and clone distributions for the methanol and methanol with humic amendments there predominance of MK7 and MK8 are clear. Hierarchical cluster analysis was performed on the respiratory quinone profiles for experiment 3 and 5. Unlike the PLFA cluster analysis, the respiratory quinones grouped by electron donor and not experiment. A second distinction is seen with methanol and perhaps with $\mathrm{pH}$. A more in-depth database on quinones from isolates and environmental samples in combination with statistical analyses will further expand the information about active respiration and the biogeochemical processes occurring in these microcosms and ultimately the ORIFC site.

\section{Conclusions:}

The microcosm experiments showed consistent findings. In most cases, all substrates promoted nitrate reduction. Glucose and ethanol promoted rapid uranium reduction. Methanol promotes $\mathrm{U}$ reduction if when certain microbial populations are present. PLFAME and quinone analyses indicated increased biomass with electron donor amendments. Changes in PLFAME and quinone community profiles were seen with the various electron donors. Methanol amendments show increased tran:cis ratio suggesting some limiting or toxic condition. It is interesting that ethanol proved to be an effective donor for rapidly reducing aqueous $\mathrm{U}(\mathrm{VI})$ along with some sediment-bound $\mathrm{U}(\mathrm{VI})$, it left much oxidized, while, essentially complete reduction of $\mathrm{U}(\mathrm{VI})$ was 
found with methanol (Madden et al.,2007). These results lead to the question of whether the presence of limiting conditions may increase the extent of $U$ reduced.

\section{Reference Cited:}

Balkwill DL, Leach FR, Wilson JT, McNabb JF, White DC. 1988. Equivalence of microbial biomass measures based on membrane lipid and cell wall components, adenosine triphosphate, and direct counts in subsurface sediments. Microbial Ecol 16:73-84.

Madden AS, Smith AC, Balkwill DL, Fagan LA, Phelps TJ. 2007. Microbial uranium immobilization independent of nitrate reduction, Env Microbiol 9(9):2321-2330.

\section{Publications and Other Products Delivered:}

The following publications and presentations were completed during the past year. TU personnel have been heavily involved in ERSP research, as evidenced by their regular attendance at ORIFC workshops and ERSP PI meetings.

\section{Publications:}

1. Schryver, J., C. Brandt, S. Pfiffner, A. Palumbo, A. Peacock, D. White, J. McKinley, and P. Long. 2006. Application of nonlinear analysis methods for identifying relationships between microbial community structure and groundwater geochemistry. Microbial Ecology 51:177-188.

2. Palumbo, A.V., J.C. Schryver, S.M. Pfiffner, T. Marsh, and C.C. Brandt. 2008. Relating microbial community structure to a dominant environmental variable in a complex environment; An example from a chromium contaminated site. pp. 61-67. In Proceedings of The 2008 International Conference on Bioinformatics and Computational Biology (Biocomp 2008) Volume I, Editors; H. R. Arabnia, M. Q. Yang, and J. Y. Yang. Las Vegas, July 14-17, 2008. CSREA Press, U.S.A

3. Baldwin, B.R. A. D. Peacock, Y-D. Gan, C. T. Resch, E. Arntzen, A. N. Smithgall, S. M. Pfiffner, B. M. Freifeld, D. C. White, and P. E. Long. 2009. In-Well Sediment Incubators to Evaluate Microbial Community Stability and Dynamics following Bioimmobilization of Uranium. Remediation J. Autumn 2009 pg. 73-89.

Presentations:

1. Pfiffner, S.M. 2008. What Do Membrane Lipids Tell Us about the Microorganisms Living in Extreme Environments? Tongji University, October 9, 2008, Shanghi, China.

2. Palumbo, A.V., J.C. Schryver, S.M. Pfiffner, T.L. Marsh, and C.C. Brandt. July 2008. Relating Microbial Community Structure to a Dominant Environmental Variable in a Complex Environment: An Example from a Chromium Contaminated Site. The 2008 International Conference on Bioinformatics \& Computational Biology. Las Vegas, NV.

3. Pfiffner, S.M., S. DiFurio, M. Gan, L.A. Fagan, A.V. Palumbo, and C.C. Brandt. April 2008. An integrated assessment of geochemical and community structure determinants of metal reduction rates in subsurface sediments. Department of Energy-Environmental Remediation Science Program PI Meeting. Leesburg, VA.

4. Palumbo, A.V., C.W. Schadt, C.C. Brant, M.S. McNeilly, A.S. Madden, H.J. Mills, D.M. Akob, L.A. Fagan, S. Bottomly, S.M. Pfiffner, and J.E. Kostka. April 2007. An Intregrated Assessment of Geochemical and Community Structure Determinants of Metal Reduction 
Rates in Subsurface Sediments. Department of Energy-Environmental Remediation Science Program PI Meeting. Leesburg, VA.

5. Vishnivetskaya, T.A., A.V. Palumbo, C.C. Brant, S.M. Pfiffner, L.A. Fagan, A.S. Madden, M.S. McNeilly, J.E. Kostka. May 2007. Variability in Community Changes in Response to Carbon Amendment for U Reduction. American Society for Microbiology. Toronto, Canada.

6. DiFurio, S., S.M. Pfiffner, L.A. Fagan, M.S. McNeilly, A.S. Madden, C.C. Brandt, and A.V. Palumbo. May 2007. PLFA Measurement of the Effects of Carbon Source Addition and Sediment Source on Uranium Reduction and Microbial Diversity Sediment Microcosms. American Society for Microbiology. Toronto, Canada.

7. Palumbo, A.V., C.W. Schadt, C.C. Brandt, J.E. Kostka, and S.M. Pfiffner. April 2006. An integrated assessment of geochemical and community structure determinants of metal reduction rates in subsurface sediments. Department of Energy-Environmental Remediation Science Program PI Meeting. Warrenton, VA. 